This item was submitted to Loughborough's Research Repository by the author.

Items in Figshare are protected by copyright, with all rights reserved, unless otherwise indicated.

\title{
Vortex matter in the charged Bose liquid at absolute zero
}

PLEASE CITE THE PUBLISHED VERSION

LICENCE

CC BY-NC-ND 4.0

\section{REPOSITORY RECORD}

Kabanov, V.V., and A.S. Alexandrov. 2019. "Vortex Matter in the Charged Bose Liquid at Absolute Zero". figshare. https://hdl.handle.net/2134/1176. 
This item was submitted to Loughborough's Institutional Repository (https://dspace.lboro.ac.uk/) by the author and is made available under the following Creative Commons Licence conditions.

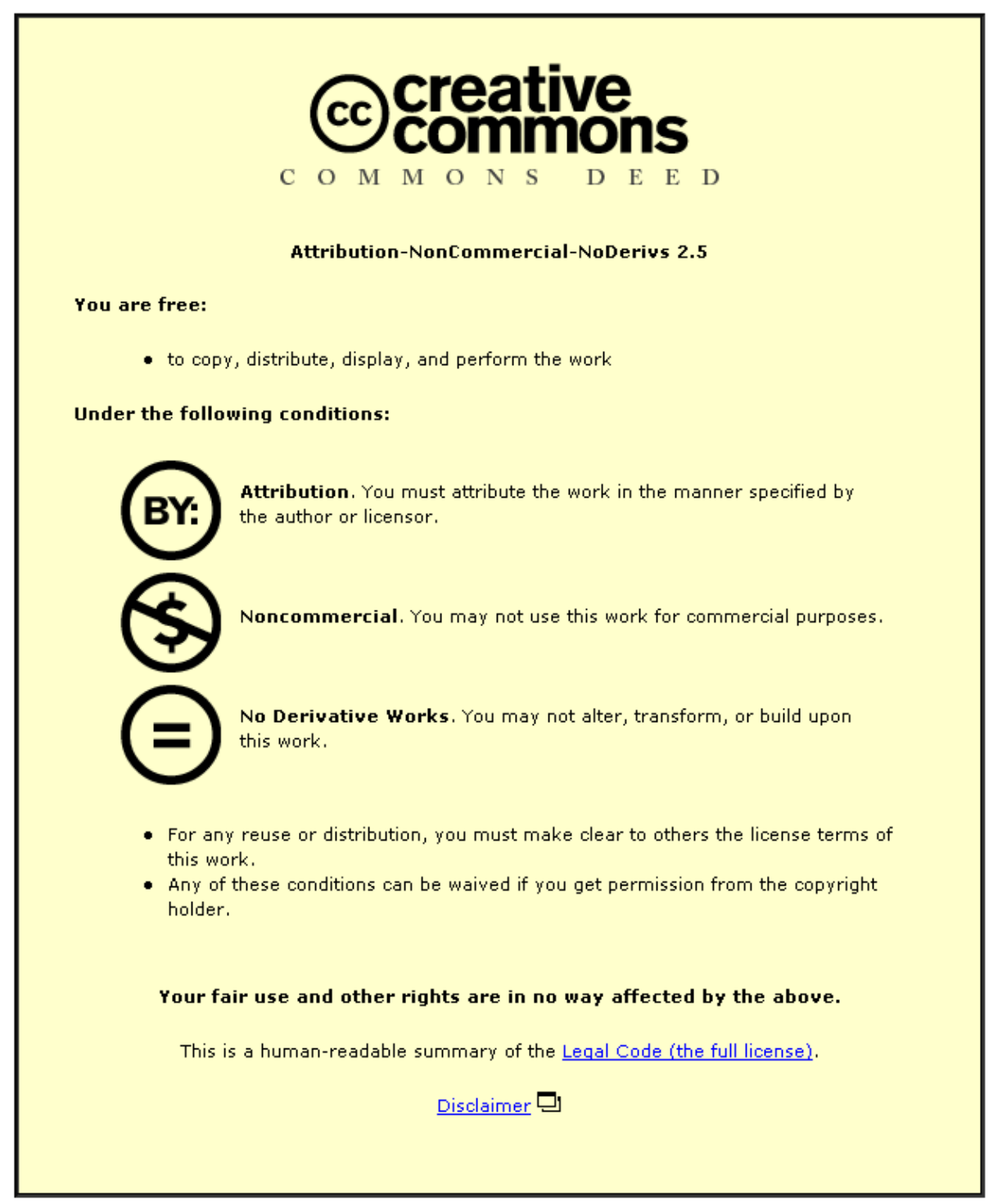

For the full text of this licence, please go to: http://creativecommons.org/licenses/by-nc-nd/2.5/ 


\title{
Vortex matter in the charged Bose liquid at absolute zero
}

\author{
V. V. Kabanov ${ }^{1}$ and A. S. Alexandrov ${ }^{2}$ \\ ${ }^{1}$ Josef Stefan Institute 1001, Ljubljana, Slovenia \\ ${ }^{2}$ Department of Physics, Loughborough University, Loughborough, United Kingdom
}

\begin{abstract}
The Gross-Pitaevskii-type equation is solved for the charge Bose liquid in an external magnetic field at zero temperature. There is a vortex lattice with locally broken charge neutrality. Remarkably, there is no upper critical field at zero temperature, so the density of single flux-quantum vortices monotonously increases with the magnetic field up to $B=\infty$ and no indication of a phase transition. The size of each vortex core decreases as about $B^{-1 / 2}$ keeping the system globally charge neutral. If bosons are composed of two fermions, a phase transition to a spin-polarized Fermi liquid at some magnetic field larger than the pair-breaking field is predicted.
\end{abstract}

PACS numbers: $74.20 .-\mathrm{z}, 74.65 .+\mathrm{n}, 74.60 . \mathrm{Mj}$

Charged Bose liquids (CBLs) have been solely of academic interest for a long time $[1,2,3,4,5,[6,7,8]$. Notwithstanding, experimental realization of the BoseEinstein condensation (BEC) of trapped ultra-cold atoms 9, 10, 11, 12, 13, 14] made it possible to create ultracold plasmas [15] by using lasers to trap and cool neutral atoms to temperatures of $1 \mathrm{mK}$ or lower. Another laser then ionizes the atoms by giving each of the outermost electrons just enough energy to escape the electrical attraction of its parent ion. The ions retain the millikelvin temperatures of the neutral atoms, so they may bose-condense, if their spin is an integer. There is also growing experimental evidence for preformed 2echarged bosons in high-temperature cuprate superconductors, such as normal state pseudogaps, unusual upper critical fields, small normal-state Lorentz numbers, etc [16]. Similar charged boson physics is expected in a lattice of mesoscale superconducting dots, if parameters are chosen such that single-electron tunnelling is suppressed and only Cooper-pair charges tunnel between the domains via Josephson tunnelling [17]. It is also possible to describe the universal features of the superconductorinsulator transition as a function of disorder in quasi-two dimensional systems in terms of boson physics 18, 19. In order to model the transition in terms of bosons, one has to include the Coulomb repulsion, otherwise all bosons would collapse into the lowest lying highly localised state.

These developments have renewed interest in CBL as a fundamental reference system. A non-interacting gas of charged bosons cannot bose-condense at any finite magnetic field because of a one-dimensional character of motion in the lowest Landau band [1]. However, interacting charged bosons condense below some (upper) critical field $B \leqslant H_{c 2}(T)$ since their collisions remove the onedimensional singularity of the density of states 20]. The BEC field diverges with decreasing temperature 20, 21], so that $H_{c 2}(T)=\infty$ at absolute zero. A single vortex in CBL has a charged core and an electric field inside [22], while its magnetic field is virtually identical to the Abrikosov vortex [23].

Here we present the ground state of CBL in an arbitrary magnetic field solving numerically the GrossPitaevskii -type equations with the long-range Coulomb interaction between bosons. We find a lattice of charged vortices, which does not disappear at any finite magnetic field. The density of vortices monotonously increases and their core size decreases with the magnetic field up to $B=\infty$. The size of vortices also depends on the thickness of CBL films different from the conventional superconducting films. When bosons are composed of two fermions, there is a phase transition to a spin-polarized Fermi liquid at some magnetic field.

The Hamiltonian of charged bosons on a compensating homogeneous background (to ensure global charge neutrality) in the external magnetic field with the vector potential $\mathbf{A}(\mathbf{r})$ is given by

$$
\begin{aligned}
H & =\int d \mathbf{r} \psi^{\dagger}(\mathbf{r})\left[-\frac{(\hbar \nabla-i e \mathbf{A} / c)^{2}}{2 m}-\mu\right] \psi(\mathbf{r}) \\
& +\frac{1}{2} \int d \mathbf{r} \int d \mathbf{r}^{\prime} V\left(\mathbf{r}-\mathbf{r}^{\prime}\right) \\
& \times\left[\psi^{\dagger}(\mathbf{r}) \psi^{\dagger}\left(\mathbf{r}^{\prime}\right) \psi\left(\mathbf{r}^{\prime}\right) \psi(\mathbf{r})-2 n \psi^{\dagger}(\mathbf{r}) \psi(\mathbf{r})\right],
\end{aligned}
$$

where $m, e, n, \mu$ are the mass, charge, average density and chemical potential of bosons, respectively, and $V(\mathbf{r})=$ $e^{2} /|\mathbf{r}|$ is their Coulomb repulsion 24].

The equation of motion for the Heisenberg field operator, $\psi(\mathbf{r}, t)$, is derived using this Hamiltonian. If the density is relatively high, so that the dimensionless Coulomb repulsion $r_{s}=m e^{2} / \hbar^{2}(4 \pi n / 3)^{1 / 3}$ is not large, one can expect that the occupation numbers of one-particle states are not very much different from those in the ideal Bosegas. In particular, one state remains macroscopically occupied at $T=0 \mathrm{~K}$. Then, following Bogoliubov 25] one separates the large matrix element $\psi_{0}$ from $\psi$ by treating the rest $\tilde{\psi}$ as small fluctuations, $\psi(\mathbf{r}, t)=\psi_{0}(\mathbf{r})+\tilde{\psi}(\mathbf{r}, t)$. The anomalous average $\psi_{0}(\mathbf{r})=\langle\psi(\mathbf{r}, t)\rangle$ is approximately equal to $\sqrt{n}$ in a homogeneous system at $T=0$. Substituting the Bogoliubov displacement transformation into the equation of motion and collecting $c-n u m b e r$ terms of $\psi_{0}$, one obtains the equation for the macroscopic condensate wave function as [22]

$$
\left[\frac{(\hbar \nabla-i e \mathbf{A} / c)^{2}}{2 m}+\mu\right] \psi_{0}(\mathbf{r})
$$



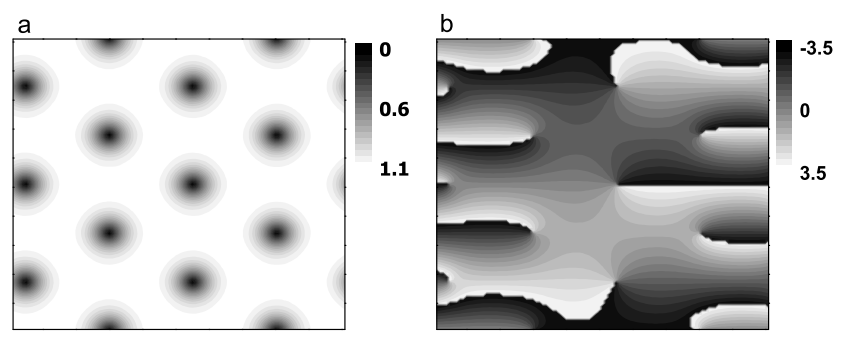

FIG. 1: A few vortices in a sample of the size $L=22.4 \xi$, (a). The phase profile of the order parameter is shown in (b). The phase changes by $2 \pi$ around each core at any magnetic field.

$$
=\int d \mathbf{r}^{\prime} V\left(\mathbf{r}-\mathbf{r}^{\prime}\right)\left[\psi_{0}^{*}\left(\mathbf{r}^{\prime}\right) \psi_{0}\left(\mathbf{r}^{\prime}\right)-n\right] \psi_{0}(\mathbf{r})
$$

The integro-differential equation (2) is quite different from original Ginzburg-Landau (GL) 26] and the GrossPitaevskii 27 equations, describing the order parameter in the BCS and neutral superfluids, respectively. As recognised by one of us 22 the coherence length in CBL is just the same as the screening radius, so the core of a single vortex is charged [28]. Indeed, introducing the dimensionless quantities: $f=\left|\psi_{0}\right| / n^{1 / 2}, \rho=\mathbf{r} / \lambda$, $\mathbf{h}=e \xi \lambda \operatorname{curl} \mathbf{A} / \hbar c$ for the order parameter, length and magnetic field, respectively, one obtains the coherence length about the same as the screening radius at $T=0 \mathrm{~K}$ [5], $\xi=\left(\hbar / 2^{1 / 2} m^{*} \omega_{p}\right)^{1 / 2}$, where $\omega_{p}=\left(4 \pi n e^{2} / m\right)^{1 / 2}$ is the zero-temperature plasma frequency [2]. The London penetration depth is conventional, $\lambda=\left(m c^{2} / 4 \pi n e^{2}\right)^{1 / 2}$, but a new feature is an electric field potential, $e \phi(\mathbf{r})=$ $\int d \mathbf{r}^{\prime} V\left(\mathbf{r}-\mathbf{r}^{\prime}\right)\left[\left|\psi_{0}\left(\mathbf{r}^{\prime}\right)\right|^{2}-n\right]$. Moreover, the chemical potential $\mu$ is zero, as it should be in the globally neutral $\mathrm{CBL}$ in the thermal equilibrium below the BEC critical temperature.

Any realistic CBL is an extreme type II with a very large Ginzburg-Landau parameter, $\kappa=\lambda / \xi \ggg 1$ [22]. For example, the coherence length and the electric field inside the vortex core are about $1 \mathrm{~nm}$ or less and $10 \mathrm{mV}$, respectively, with the material parameters typical for cuprates $\left(m=10 m_{e}, n=10^{21} \mathrm{~cm}^{-3}\right.$ and $\left.\epsilon_{0} \gtrsim 100\right)$, and $\kappa$ is about $10^{3}$ with these parameters. Hence, the magnetic field is practically homogeneous, and the ground state $\psi_{0}(\mathbf{r})$ can be found by minimizing the energy functional $E$ with respect to $\psi_{0}(\mathbf{r})$,

$$
\begin{gathered}
E\left(\psi_{0}\right)=\frac{1}{2 m} \int d \mathbf{r} \mid(\hbar \nabla-i e \mathbf{A} / c) \psi_{0}\left(\left.\mathbf{r}\right|^{2}\right. \\
+\frac{1}{2} \int d \mathbf{r} \int d \mathbf{r}^{\prime} V\left(\mathbf{r}-\mathbf{r}^{\prime}\right)\left|\psi_{0}(\mathbf{r})\right|^{2}\left(\left|\psi_{0}\left(\mathbf{r}^{\prime}\right)\right|^{2}-2 n\right),
\end{gathered}
$$

where $\mathbf{A}=\{0, B x, 0\}$. In numerical simulations we consider a sample with the rectangular cross-section $L \times L$ and the magnetic flux $B L^{2}=m \Phi_{0}$, where $m$ is an integer ( $\Phi_{0}$ is the flux quantum). When the magnetic field $\mathbf{B}$ is applied along z-direction, the order parameter $\psi_{0}(x, y)$ does not depend on $z$ obeying the following translation
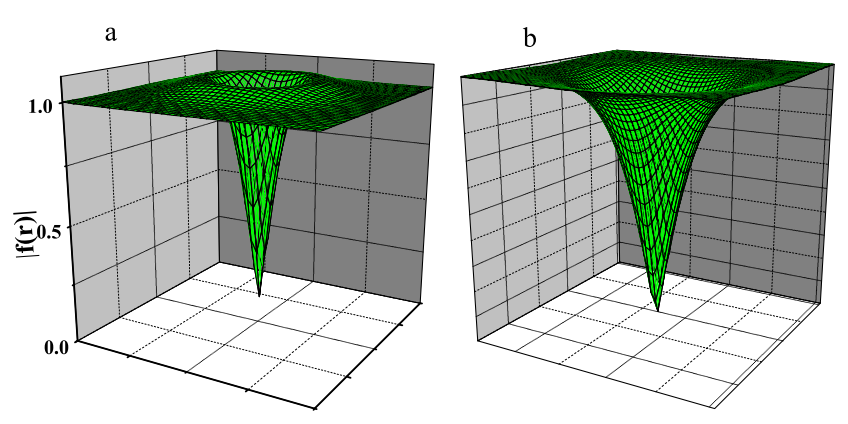

FIG. 2: Single vortex in CBL 22] (a) compared with the Abrikosov vortex (b).

symmetry,

$$
\begin{aligned}
& \psi_{0}(x+L, y)=\exp (-i e B L y / \hbar c) \psi_{0}(x, y) \\
& \psi_{0}(x, y+L)=\psi_{0}(x, y)
\end{aligned}
$$

These relations can be used as boundary conditions when $\mathrm{m}$ is an integer.

Because Eq.(3) does not contain the penetration depth, it is convenient to introduce new dimensionless coordinates $\mathbf{x}=\mathbf{r} / \xi$, the vector potential, $\mathbf{a}=$ $\left(0,2 \pi B x \xi^{2} / \Phi_{0}, 0\right)$, and the Coulomb energy $v(\mathbf{x})=$ $e \phi /\left(\omega_{p}^{2} m^{*} \xi^{2}\right)$. As a result, the problem is reduced to minimization of the functional

$$
\begin{aligned}
& E(f)=\frac{\hbar^{2} n \xi}{2 m} \int d \mathbf{x}\left[|(\nabla-i \mathbf{a}) f(\mathbf{x})|^{2}\right. \\
+ & \left.v(\mathbf{x})\left(|f(\mathbf{x})|^{2}-1\right)\right]
\end{aligned}
$$

where the Coulomb field satisfies the Poisson equation,

$$
\Delta v(\mathbf{x})=1-|f(\mathbf{x})|^{2} .
$$

To compare CBL vortex state with the Abrikosov vortex lattice we also minimize the conventional GL functional using the same dimensionless unites,

$$
\begin{aligned}
& E_{G L}(f)=\frac{\hbar^{2} n_{s} \xi}{2 m} \int d \mathbf{x}\left[|(\nabla-i \mathbf{a}) f(\mathbf{x})|^{2}\right. \\
& \left.-|f(\mathbf{x})|^{2}+\frac{1}{2}|f(\mathbf{x})|^{4}\right]
\end{aligned}
$$

where $\xi=\hbar^{2} /(2 m|\alpha|)^{1 / 2}, n_{s}=|\alpha| / \beta$ and the order parameter $f$ is normalised by $\sqrt{n_{s}}$. Here $\alpha$ and $\beta$ are conventional GL coefficients [26]. We apply the standard discretization procedure described in Ref.[29]. Eq.(6) for the electrostatic potential is solved by the Fourier transform in the discrete form, and the resulting energy is minimized with the conjugated gradient algorithm.

Since both functionals depend only on the dimensionless vector-potential a which is proportional to the product $B \xi^{2}$, simulations can be performed at fixed $L$ and $\xi$ by changing $B$ or at fixed $L$ and $B$ by changing $\xi$. Our numerical results are shown in Figs. 1-4. At any value of the magnetic field we find the triangular vortex lattice. 


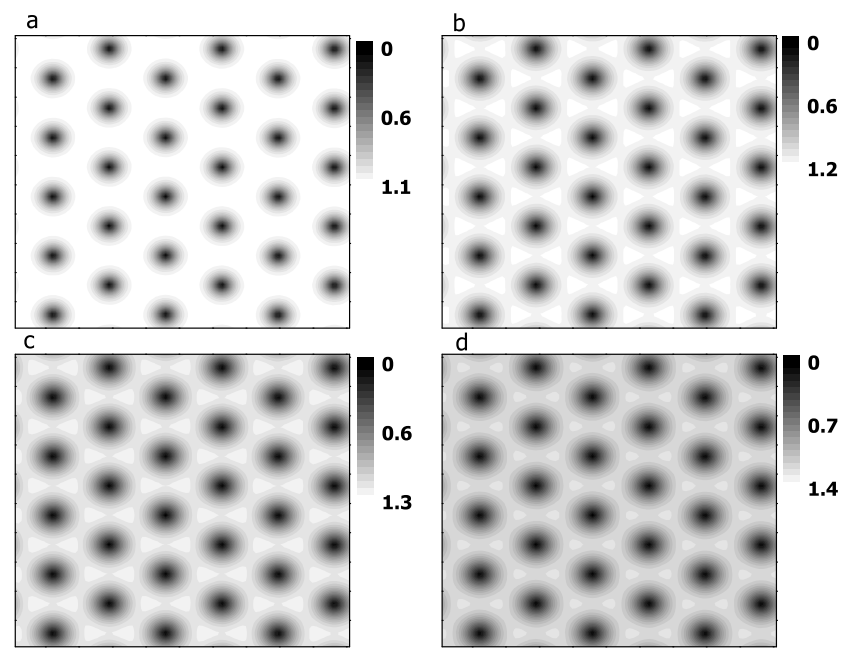

FIG. 3: The vortex lattice in CBL for 30 flux quantum per cross-section, (a) $L / \xi=33.67$, (b) $L / \xi=25.25$, (c) $L / \xi=14.43$, (d) $L / \xi=10.1$ One can see from the scale near each figure that the order parameter remains large outside the cores, $f>1$ at any $\xi$ (or at any magnetic field).

While the field is small, there are only a few vortices per sample cross-section, Fig.1a. When vortices are far apart, their interaction yields only a small contribution to the energy functional but even in that case a triangular lattice of vortices is clearly seen in CBL, Fig.1.

Each vortex carries one flux quantum, as can be seen from the phase profile in Fig.1b. It has an unusual core, Fig.2, in agreement with Ref. 22], which differs qualitatively from the Abrikosov vortex [23] due to a local charge redistribution caused by the magnetic field. The breakdown of the local charge neutrality, Fig.2, is due to the absence of an equilibrium normal state solution in CBL at $T=0$ with $\psi_{0}=0$, as explained in Ref. 22].

Increasing the field first increases the vortex density with about constant size of the cores, as in conventional superconductors, Fig.3 and Fig.4. However, quite different from the Abrikosov lattice, increasing the field further does not lead to a superfluid to normal phase transition, but instead it increases the density of vortices by decreasing the size of every individual core, Fig.3c,d. Remarkably, each vortex carries one flux quantum at any field. Keeping the global charge neutrality the charge heterogeneity depends on the magnetic field, and the core diameters decrease with the field, when the field is large, $\xi^{2}>2 \pi \hbar c /(e B)$. Indeed, in this regime the "bare" coherence length $\xi$ becomes irrelevant, but the only characteristic length is the distance between single flux-quantum vortices, i.e. $r \approx \sqrt{2 \pi \hbar c /(e B)}$. As a result, the amplitude real-space modulations of the order parameter increase with the magnetic field in CBL, while they decrease in conventional superconductors, where the order parameter vanishes at and above the finite $H_{c 2}=\Phi_{0} /\left(2 \pi \xi^{2}\right)$ (fig. $\left.4 \mathrm{c}, \mathrm{d}\right)$.

There is another difference between CBL and conven-

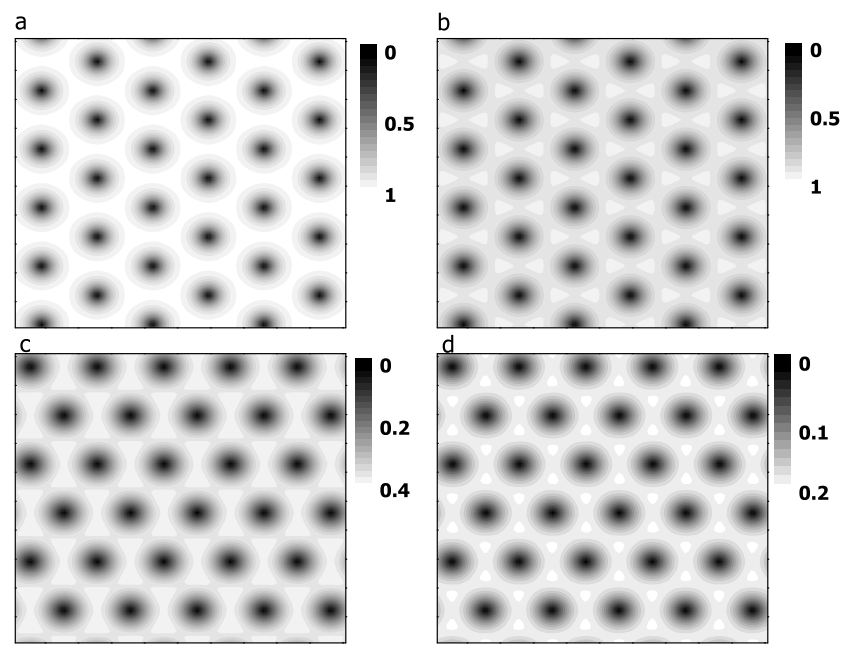

FIG. 4: The Abrikosov vortex lattice for 30 flux quantum per cross-section (a) $L / \xi=33.67$, (b) $L / \xi=25.25$, (c) $L / \xi=$ 14.43 , and for (c) $L / \xi=13.87$, which corresponds to $B$ close to $H_{c 2}=\Phi_{0} /\left(2 \pi \xi^{2}\right)$. The order parameter decreases when $B$ approaches the conventional upper critical field.

tional vortex matter in case of thin films. If we assume that the film thickness $d$ is small, $d \ll \xi$, then the left hand side of Eq.(6) takes the form $\left(1-|f(\mathbf{x})|^{2}\right) d \delta(z) / \xi$. The dimension analysis readily shows that the true coherence length, $\xi_{2 D}$ depends on the thickness as $\xi_{2 D}=$ $\left(\xi^{4} / d\right)^{1 / 3}$ in that case. As a result the size of vortex cores depends on the thickness of CBL films different from the conventional films.

There is also an important consequence of the infinite (orbital) upper critical field at absolute zero in such CBLs, where singlet bosons are formed of two fermions [16]. In this case sufficiently large magnetic field can break bound pairs via a spin-flip of one of two fermions, if triplets are unstable, because the singlet binding energy $\Delta$ decreases with the field as $\Delta(B)=\Delta-2 \mu_{B} B$ $\left(\mu_{B}=e \hbar /\left(2 m_{e}\right)\right.$ is the electron Bohr magneton) [30]. A spin-polarised Fermi liquid appears at $B \geqslant H_{p}$, where $H_{p}=\Delta /\left(2 \mu_{B}\right)$ is the pair-breaking field. In this estimate we neglect the orbital (Landau) diamagnetism of bosons and fermions, and the Coulomb energy of the chargedmodulated vortex lattice. The latter is of the order of $e \phi_{c} n \xi^{2} B / \Phi_{0}$ per unit volume, where $\phi_{c} \sim \hbar^{2} /\left(e m \xi^{2}\right)$ is the characteristic electrostatic potential inside vortex cores. The Coulomb energy is small compared with the spin (Pauli) contribution if $m_{e} / m \ll 1$, which we assume to be the case, so diamagnetic contributions are also small. However, bound pairs still survive up to a higher field $H^{*}=H_{p}+n /\left(N \mu_{B}\right)>H_{p}$ due to the Pauli exclusion principle, which prevents any further decay of pairs, if the number of fermions $\approx N|\Delta(B)|$ remains smaller than $2 n$ ( $N$ is the fermion density of states). There is a boson-fermion mixture, if $H_{p}<B<H^{*}$, with the fermion density modulated in real space because of charged vortices. Normal fermions (as well as normal 
bosons pushed up from the condensate by temperature) are distributed inhomogeneously across the sample with the maximum density in the vortex cores, where their potential energy is at minimum. The excess density of normal carriers inside the cores screens the electric field caused by the inhomogeneous condensate density. If the screening length due to normal fermions becomes smaller than the coherence length $\xi$, one can expect a nontrivial field dependence of the size of vortices, which disappear at $B=H^{*}$.

In conclusion, we have found the triangular lattice of single-flux-quantum charged vortices in CBL which cannot be destroyed by any magnetic field at zero temperature. The vortex density monotonously increases and their core size decreases with the magnetic field up to $B=\infty$ with no indication of a phase transition. The core size depends on the thickness of CBL films. At finite temperatures $H_{c 2}(T)$ is finite [20, 21]. Neverthe- less, unusually large charge modulations with the scale depending on the magnetic field should persist at finite temperatures as well. The phase transition to the spinpolarized Fermi liquid at some magnetic field larger than the pair-breaking field has been predicted for preformed bosonic pairs. These results are relevant for real charged Bose-liguids in ultracold plasmas and in the superconducting cuprates, and for superconducting quantum dots and superconductor-insulator phase transitions described by a similar boson physics. There is also a close analogy between the vortex structure in CBL and the Josephson vortices. Since the normal phase is not defined below $T_{c}$, there is no "normal" vortex core in CBL, and there is no "normal" core in the Josephson vortex either. One can define the lower critical field $H_{c 1}$ when a first vortex penetrates into CBL 22] and into the Josephson junction 31], but the upper critical field is infinite in both cases.
[1] M.R. Schafroth, Phys.Rev. 100, 463 (1955).

[2] L.L. Foldy, Phys.Rev. 124, 649(1961).

[3] A.L. Fetter, Ann. Phys. (N.Y.) 64,1 (1971).

[4] R.F. Bishop, J.Low Temp.Phys. 15, 601(1974).

[5] S.R. Hore and N.E. Frankel, Phys.Rev. B 12, 2619(1975); ibid 14, 1952(1976).

[6] D.K. Lee and E. Feenberg, Phys.Rev. 137, A731 (1965).

[7] K.A. Brueckner, Phys. Rev. 156, 204 (1967).

[8] C-W. Woo and S. Ma, Phys.Rev. 159, 176 (1967).

[9] M.H. Anderson et al., Science 269, 198 (1995).

[10] K.B. Davis et al., Phys. Rev. Lett. 75, 3969 (1995).

[11] C.C. Bradley, C.A. Sackett, J.J. Tollett,and R.G. Hulet, Phys. Rev. Lett. 75, 1687 (1995).

[12] M.-O. Mewes et al., Phys. Rev. Lett. 78, 582 (1997).

[13] E.W. Hagley et al., Science 283, 1706 (1999).

[14] A.G. Truscott et al., Science 291, 2570 (2001); F. Schreck et al., Phys. Rev. Lett. 87, 080403 (2001); S.R. Granade, M.E. Gehm, K.M. OHara, and J.E. Thomas, Phys. Rev. Lett. 88, 120405 (2002).

[15] T.C. Killian, S. Kulin, S. D. Bergeson, L. A. Orozco, C. Orzel, and S. L. Rolston, Phys. Rev. Lett. 83, 4776 (1999); S. Bergeson and T. Killian, Physics World 16, 37, February (2003).

[16] A.S. Alexandrov, Theory of Superconductivity: from Weak to Strong coupling, (IoP Publishing, BristolPhiladelphia, 2003) and references therein.

[17] M.P.A. Fisher, G. Grinstein, and S.M. Girvin, Phys. Rev. Lett. 64, 587 (1990); for a review of Josephson-junction circuits see Yu. Makhlin, G. Schön, and A. Shnirman, in New Directions in Mesoscopic Physics, eds. R. Fazio, V.F. Gantmakher, and Y. Imry (Kluwer, 2003).

[18] M. P. A. Fisher, P. B. Weichman, G. Grinstein and D. S. Fisher, Phys. Rev. B 40, 546 (1989).

[19] M. Wallin, E. S. Srensen, S. M. Girvin, and A.P. Young,
Phys. Rev. B49, 12115 (1994).

[20] A.S. Alexandrov, Doctoral thesis MEPHI (Moscow) (1984); A.S. Alexandrov, Phys.Rev. B48, 10571 (1993).

[21] A.S. Alexandrov, W.H. Beere, and V.V. Kabanov, Phys. Rev. B54, 15363 (1996).

[22] A.S. Alexandrov, Phys. Rev. B60, 14573 (1999).

[23] A.A. Abrikosov, Zh. Eksp. Teor. Fiz. 32, 1442 (1957) ( Soviet Phys. JETP 5, 1174 (1957)).

[24] The Coulomb repulsion of preformed pairs is $4 e^{2} / r \epsilon_{0}$, where $\epsilon_{0}$ is the static dielectric constant of the host material and $e$ is the elementary charge.

[25] N. Bogoliubov, J.Phys. USSR 11, 23-32(1947).

[26] V.L. Ginzburg and L.D. Landau, Zh. Eksp. Teor. Fiz. 20, 1064 (1950).

[27] E.P. Gross, Nuovo Cimento 20, 454 (1961); L.P. Pitaevskii, Zh. Eksp. Teor. Fiz. 40, 646 (1961) ( Soviet Phys. JETP 13, 451 (1961)).

[28] Charging effects are very small in conventional superconductors because the screening radius is much smaller than the coherence length (D.I. Khomskii and A. Freimuth, Phys. Rev. Lett. 75, 1384 (1995); G. Blatter, M. Feigelman, V. Geshkenbein, A. Larkin, and A. vanOtterlo, Phys. Rev. Lett. 77, 566 (1996)); E. Babaev, Phys. Rev. B63, 172502 (2001)).

[29] J. Bonca, V.V. Kabanov, Phys. Rev. B, 65, 012509, (2002); T. Mertelj, V.V. Kabanov, ibid, 67, 134527, (2003).

[30] V.N. Zavaritsky, M. Springford and A.S. Alexandrov, EuroPhys. Lett. 51, 334 (2000).

[31] V.V. Schmidt, The Physics of Superconductors: Introduction to Fundamentals and Applications, (SpringerVerlag, Berlin, 1997). 